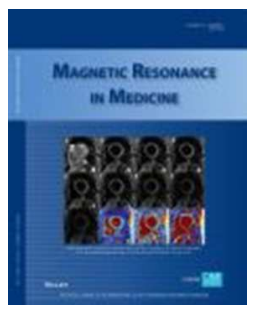

\title{
Improved Liver R2* Mapping by Pixel-wise Curve Fitting with Adaptive Neighborhood Regularization
}

\begin{tabular}{|c|c|}
\hline Journal: & Magnetic Resonance in Medicine \\
\hline Manuscript ID & MRM-17-18015.R1 \\
\hline Wiley - Manuscript type: & Full Paper \\
\hline Date Submitted by the Author: & $\mathrm{n} / \mathrm{a}$ \\
\hline Complete List of Authors: & $\begin{array}{l}\text { Wang, Changqing; University of Electronic Science and Technology of } \\
\text { China, School of Automation Engineering; Southern Medical University, } \\
\text { School of Biomedical Engineering; University of Wisconsin-Madison, } \\
\text { Department of Radiology } \\
\text { Zhang, Xinyuan; Southern Medical University, School of Biomedical } \\
\text { Engineering } \\
\text { Liu, Xiaoyun; University of Electronic Science and Technology of China, } \\
\text { School of Automation Engineering } \\
\text { He, Taigang; St George's University of London, Cardiovascular Science } \\
\text { Research Centre; Royal Brompton Hospital, CMR Unit } \\
\text { Chen, Wufan; University of Electronic Science and Technology of China, } \\
\text { School of Automation Engineering; Southern Medical University, School of } \\
\text { Biomedical Engineering } \\
\text { Feng, Qianjin; Southern Medical University, School of Biomedical } \\
\text { Engineering } \\
\text { Feng, Yanqiu; Southern Medical University, School of Biomedical } \\
\text { Engineering }\end{array}$ \\
\hline Research Type: & $\begin{array}{l}\text { T2* < Relaxation techniques }<\text { Technique Development }<\text { Technical } \\
\text { Research }\end{array}$ \\
\hline Research Focus: & Liver $<$ Abdominal/Pelvic \\
\hline
\end{tabular}

\section{SCHOLARONE ${ }^{\text {M }}$}

Manuscripts 
Improved Liver R2* Mapping by Pixel-wise Curve Fitting with Adaptive Neighborhood Regularization

Changqing Wang ${ }^{1,2,3}$, Xinyuan Zhang ${ }^{2}$, Xiaoyun Liu ${ }^{1}$, Taigang $\mathrm{He}^{4,5}$, Wufan Chen ${ }^{1,2}$, Qianjin Feng ${ }^{2}$, and Yanqiu Feng ${ }^{2 *}$

1. School of Automation Engineering, University of Electronic Science and Technology of China, Chengdu, China

2. School of Biomedical Engineering, Southern Medical University, Guangzhou, China

3. Department of Radiology, University of Wisconsin, Madison, Wisconsin, USA

4. Cardiovascular Sciences Research Centre, St George's University of London, London, United Kingdom

5. Royal Brompton Hospital and Imperial College, London, United Kingdom

\section{Corresponding author:}

Dr. Yanqiu Feng $\quad$ Email: foree@163.com

School of Biomedical Engineering,

Southern Medical University,

Guangzhou, China

Tel. +862061648271

Fax +86 2061648274

Running title: R2* Mapping with Adaptive Neighborhood Information

The word count: 3915

Key words: MR relaxometry; hepatic iron concentration; non-central chi noise; adaptive neighborhood regularization; R2* mapping 


\begin{abstract}
Purpose: To improve liver R2* mapping by incorporating adaptive neighborhood regularization into pixel-wise curve fitting.

Methods: MRI R2* mapping remains challenging due to the serial images with low signal-to-noise ratio (SNR). In this study, we proposed to exploit the neighboring pixels as regularization terms and adaptively determine the regularization parameters according to the inter-pixel signal similarity. The proposed algorithm, termed as pixel-wise curve fitting with adaptive neighborhood regularization (PCANR), was compared with the conventional non-linear least squares (NLS) and non-local means filter-based NLS (NLM-NLS) algorithms on simulated, phantom, and in vivo data.

Results: Visually, the PCANR algorithm generates R2* maps with significantly reduced noise and well-preserved tiny structures. Quantitatively, the PCANR algorithm produces R2* maps with lower root mean square errors at varying R2* values and SNR levels compared with the NLS and NLM-NLS algorithms. For the high R2* values under low SNR levels, the PCANR algorithm outperforms the NLS and NLM-NLS algorithms in the accuracy and precision, in terms of mean and standard deviation of $\mathrm{R} 2 *$ measurements in selected region of interest respectively.

Conclusions: The PCANR algorithm can reduce the effect of noise on liver R2* mapping, and the improved measurement precision will benefit the assessment of hepatic iron in clinical practice.
\end{abstract}

Key words: MR relaxometry; hepatic iron concentration; non-central chi noise; adaptive neighborhood regularization; R2* mapping 


\section{INTRODUCTION}

Chronic blood transfusions might induce excessive iron deposition in patients with transfusion-dependent anemia, such as thalassemia major and sickle cell disease. A timely and accurate iron chelation therapy is needed to maintain the body iron at safe levels while minimizing the risks of toxicity from iron chelation (1). Considering that excess iron tends to accumulate in the liver, hepatic iron concentration (HIC) is commonly used as a surrogate for the total body iron loading $(2,3)$. Percutaneous liver biopsy is the current gold standard for the evaluation of HIC. However, liver biopsy is an invasive, painful, and expensive procedure with $1 \%-4 \%$ risk of potentially serious complications (4,5). In addition, notable deviations might be incurred from the sampling errors caused by the sample size and heterogeneous distribution of hepatic iron (6-9). These limitations can be avoided by applying MRI-based iron quantification methods $(10,11)$. The transverse relaxation time (T2) and effective transverse relaxation time $(\mathrm{T} 2 *)$ techniques have emerged as reliable alternatives, and the relationships between R2 (1/T2) or R2* (1/T2*) and biopsied HIC have been well described (12,13).

MRI R2* technique is becoming popular in clinical practice partly due to the short imaging time. For the liver R2* measurement, a representative value is typically obtained to assess the liver iron loading. Compared with the R2* measurement based on the expectations of the measured signals within a region of interest (ROI), the R2* mapping by pixel-wise curve fitting has the advantage of depicting the spatial distribution of HIC (12-15), which might reveal physiologically relevant information. However, R2* mapping remains challenging due to the low signal-to-noise ratio (SNR) serial images, especially in scenarios of high HIC. Multichannel array coils are usually applied, and MRI data are reconstructed by root-sum-square operation to obtain high SNR serial images. In such scenarios, the noise is assumed to follow a non-central chi distribution (16). Several noise-corrected curve fitting models were developed to address the noise issue $(17,18)$. We have recently demonstrated that 
fitting the signal to its first and second moments in the presence of non-central chi noise $\left(\mathrm{M}^{1} \mathrm{NCM}\right.$ and $\left.\mathrm{M}^{2} \mathrm{NCM}\right)$ are preferable for the ROI-based R2* estimation. The R2* map generated by pixel-wise curve fitting using the $\mathrm{M}^{1} \mathrm{NCM}$ model-based non-linear least squares (NLS) algorithm is still noisy for severe iron-overloaded liver (19).

Recently, exploiting neighborhood similarity with low-rank and/or sparsity constraints is promising in accurately estimating parameter maps from undersampled MRI data (20-22). With regard to R2* mapping, a neighborhood exploiting approach, which combines the non-local means (NLM) denoising filter (23) and the $\mathrm{M}^{2} \mathrm{NCM}$ model, was shown to effectively reduce the effect of noise on quantified parameter maps (24). The preliminary results of combining the NLM filter and the $\mathrm{M}^{1} \mathrm{NCM}$ model were also presented in an early conference paper (25). However, in such two-step approaches, the error induced in the pre-filtering step might propagate to the following curve fitting step.

To further improve the performance of noise suppression in R2* mapping, we proposed a novel method termed as pixel-wise curve fitting with adaptive neighborhood regularization (PCANR). In this algorithm, the neighboring pixels were exploited as regularization terms, and the regularization parameters were adaptively determined according to the inter-pixel signal similarity. Simulation, phantom, and in vivo experiments were conducted to evaluate the performance of the proposed method. The results of the proposed method were compared with those using the conventional NLS and two-step NLM-NLS algorithms.

\section{METHODS}

\section{R2* Quantification}

By using the $\mathrm{M}^{1} \mathrm{NCM}$ model, R2* can be measured with high accuracy and precision, which is comparable to the best achievable precision defined by the Cramer-Rao 
lower bound $(17,18)$. The $\mathrm{M}^{1} \mathrm{NCM}$ model was therefore adopted for the NLS curve fitting in this study. As shown in (18), the first moment, i.e., the expectation of measured signal $S_{M}$, in the presence of non-central chi noise is formulated as:

$$
E\left(S_{M}\right)=\sigma_{g} \sqrt{\frac{\pi}{2}} \frac{\left(2 N_{R C}-1\right) ! !}{2^{N_{R C}-1}\left(N_{R C}-1\right) !} \quad{ }_{1} F_{1}\left(-\frac{1}{2} ; N_{R C} ;-\left(\frac{s}{\sqrt{2} \sigma_{g}}\right)^{2}\right)
$$

where $\sigma_{g}$ is the standard deviation (SD) of Gaussian white noise in each channel and is estimated from the background area (18); $N_{R C}$ denotes the number of receiver coils; $S$ is the monoexponential decay signal free of noise $\left(S=S_{0} \cdot \exp \left(-T E \cdot R 2^{*}\right)\right.$, where $S_{0}$ denotes the signal intensity at zero TE); !! is the double factorial and ${ }_{1} F_{1}$ is the confluent hypergeometric function. Given that the direct calculation of the confluent hypergeometric function is highly time consuming, the approximation by a rapid look-up table method was used for fast realization as previously described (19).

\section{Conventional NLS Algorithm}

In the R2* mapping, the decay signals at each pixel were fitted to derive the corresponding R2* value by minimizing the following objective function:

$$
\min _{S_{0}, R 2^{*}}\left\|S_{x_{i}}-f\left(S_{0}, R 2^{*}\right)\right\|_{2}^{2}, \forall x_{i} \in I
$$

where $S_{x_{i}}$ is a vector representing the intensities of decay signals at target pixel $x_{i}$ in image domain $I$, and $f(\cdot)$ is the curve fitting model, which was selected as $\mathrm{M}^{1} \mathrm{NCM}$ model (right-hand side of Eq. [1]) in this study. A nonlinear Levenberg-Marquardt optimization was implemented to solve Eq. [2] $(26,27)$.

\section{NLM-NLS Algorithm}

As described in $(24,25)$, the NLM-NLS algorithm filters the serial images using the NLM filter (23) and subsequently fits the filtered signals pixel-wisely using Eq. [2] to obtain the R2* map. The serial images were separately filtered, and the filtered output 
at each target pixel was the weighted average of all the pixels in the search window. The weight was adaptively calculated based on the Gaussian-weighted Euclidean distance between the neighborhood patches of the target pixel and its neighboring pixel; large weights were assigned to the pixels with similar neighboring patches. Given the filtered images, R2* map can be obtained by the conventional NLS algorithm (Eq. [2]).

\section{PCANR Algorithm}

Pixels with similar decay signals can be assumed to have similar R2* values, and thus can be simultaneously fitted to reduce the effect of noise. The PCANR algorithm was given by minimizing the following objective function:

$$
\begin{aligned}
\min _{S_{0}, R 2^{*}}\left\|S_{x_{i}}-f\left(S_{0}, R 2^{*}\right)\right\|_{2}^{2} & \\
& +\sum_{x_{j} \in \Omega_{i}, j \neq i} \lambda\left(x_{i}, x_{j}\right)\left\|S_{x_{j}}-f\left(S_{0}, R 2^{*}\right)\right\|_{2}^{2}, \forall x_{i} \in I
\end{aligned}
$$

where the first part is the fidelity term, and the second part is the regularization term. $x_{j}$ is the neighboring pixel in search window $\Omega_{i}$ around target pixel $x_{i}$, the regularization parameter $\lambda\left(x_{i}, x_{j}\right)$ was adaptively calculated as follows:

$$
\lambda\left(x_{i}, x_{j}\right)=\exp \left(-\frac{\left\|S_{x_{i}}-S_{x_{j}}\right\|_{2}^{2}}{h^{2}}\right), \quad \forall x_{j} \in \Omega_{i} \text { and } x_{j} \neq x_{i},
$$

where parameter $h$ controls the degree of smoothing. This parameter was related to the noise level and was determined by $h=\beta \sigma_{g}$, where $\beta$ is a tuning parameter. During implementation, $\lambda\left(x_{i}, x_{j}\right)$ was normalized by its maximum. Every neighboring pixel $x_{j} \in \Omega_{i}$ contributed to the R2* estimation for the target pixel $x_{i}$. Its contribution was controlled by the regularization parameter $\lambda\left(x_{i}, x_{j}\right)$, which can be calculated based on the Euclidean distance $\left(\|\cdot\|_{2}\right.$ in Eq. [4]). The regularization parameter $\lambda\left(x_{i}, x_{j}\right)$ is large when the decay signals at pixel $x_{j}$ are similar to the decay signals at pixel $x_{i}$, whereas this parameter is small when the decay signals at 
pixel $x_{j}$ are dissimilar to those at pixel $x_{i}$.

Figure 1 shows the regularization parameters for pixels in a search window centered at a representative parenchyma pixel and the corresponding decay signals. The target pixel was located in the parenchyma adjacent to vessels. Figure $1 \mathrm{~d}$ shows that the parenchyma pixels in the search window are assigned with large regularization parameters, whereas the vessel pixels in the search window are assigned small regularization parameters. Figure 1e shows the decay signals at multiple TEs for all the pixels in the search window. The pixels with large regularization parameters have decay signals similar to the target pixel.

\section{Parameter Settings}

The parameter settings (search window $\Omega_{i}$ and smoothing parameter $h$ ) in the PCANR algorithm are of vital importance for the performance of R2* mapping. For a good trade-off between the computational load and R2* mapping performance, the search window $\Omega_{i}$ was empirically set to $11 \times 11$ for both the NLM-NLS and PCANR algorithms in this study. The influence of smoothing parameter $h$ on R2* mapping was first evaluated by simulation and an approximately optimal $h$ value was then determined. A fixed smoothing parameter $h$ of $2.0 \sigma_{g}$ was used for the PCANR algorithm in the following simulation, phantom, and in vivo studies. With regard to the NLM-NLS algorithm, the optimal $h$ that produced minimum RMSE value was used in the simulation study, whereas a fixed $h$ of $3.0 \sigma_{g}$ was used in the phantom and in vivo studies.

\section{Experimental Data}

Simulations

Magnetic Resonance in Medicine 
A mask delineating liver anatomy (Fig. 1a, including two compartments: parenchyma and blood vessels) and a non-uniform $S_{0}$ reference map (Fig. 1b) were derived from an in vivo liver dataset through a semiautomatic parenchyma extraction method (28). Both the mask with complicated structures and the non-uniform $S_{0}$ map were designed to mimic a realistic liver. In the simulations, the liver parenchyma $\mathrm{R} 2 *$ values ranged from $100 \mathrm{~s}^{-1}$ to $1000 \mathrm{~s}^{-1}$ with an increment of $100 \mathrm{~s}^{-1}$, and the vessels $\mathrm{R} 2 *$ values were constantly set to $33 \mathrm{~s}^{-1}$. Figure 1c shows an example of R2* map with liver parenchyma R2* value of $500 \mathrm{~s}^{-1}$. The noise-free image for each channel was generated by sampling the monoexponential decay model at the TEs that correspond to the actual settings for in vivo experiments (see below). Noisy images were then synthesized by adding zero mean Gaussian complex noise with SD $\sigma_{g}$ to the noise-free images from each channel and performing the root-sum-square operation of all channels. Note that the number of receiver coils $N_{R C}$ was set to 8, same as the in vivo study. Noisy images were simulated with SNRs of 15, 30, and 60 to evaluate the performance of R2* mapping under varying noise levels; SNR was defined as $\max \left(S_{0}\right) / \sigma_{g}$ to avoid dependence on TE.

To evaluate the performance of the proposed algorithm with varying R2* values, a R2* map was synthesized using a Gaussian function with a SD of 24 pixels to model slowly-varying iron overload levels (Fig. 6a). Mean R2* value for the liver parenchyma was $800 \pm 160 \mathrm{~s}^{-1}$; and the liver vessel R2* values were constantly set to $33 \mathrm{~s}^{-1}$ for simplicity. Simulations were then performed with SNRs of 15, 30, and 60 in the aforementioned way.

\section{Phantom Data}

A phantom was designed with eight agar-based vials, and each vial was filled with distilled water and different concentrations of iron (Ferumoxytol, AMAG Pharmaceuticals, Lexington, MA), ranging from $0 \mu \mathrm{g} / \mathrm{ml}$ to $441.18 \mu \mathrm{g} / \mathrm{ml}$. All vials 
were submerged in a tap water bath and scanned using a 3D multi-echo spoiled gradient echo pulse sequence on a 1.5 Tesla (T) clinical MRI system (GE Healthcare, Waukesha, WI) with an eight-channel phased-array coil. The imaging parameters were set as follows: repetition time $(\mathrm{TR})=12.98 \mathrm{~ms}, \mathrm{TE}_{\min }=0.92 \mathrm{~ms}$, echo spacing $=$ $0.8 \mathrm{~ms}$, number of echoes $=12$, flip angle $=2^{\circ}$ to minimize T1-related bias, slice thickness $=3 \mathrm{~mm}$, number of excitations $(\mathrm{NEX})=1$, matrix size $=256 \times 256$, and field of view $=200 \times 400 \mathrm{~mm}^{2}$. The phantom was scanned 16 times with the same protocol and was averaged to assess the influence of SNR on R2* mapping.

\section{In Vivo Data}

The data from four subjects (two females and two males, ages $22 \pm 8$ ) with iron overload from normal to severe were retrospectively analyzed after the approval from our institutional review board and the informed consent were obtained. The patients were performed on a 1.5 Tesla (T) Sonata scanner (Siemens Medical Solutions, Erlangen, Germany) using a six-channel anterior array coil combined with a two-channel spine array coil and 2D spoiled gradient echo acquisition with fat saturation. Axial images were acquired with the following parameters: TR $=200 \mathrm{~ms}$, $\mathrm{TE}_{\min }=0.93 \mathrm{~ms}$, echo spacing $=1.34 \mathrm{~ms}$, number of echoes $=12$, flip angle $=20^{\circ}$, slice thickness $=10 \mathrm{~mm}, \mathrm{NEX}=1$, matrix size $=64 \times 128$, and field of view $=200 \times$ $400 \mathrm{~mm}^{2}$. All echoes were acquired in a single TR using monopolar readouts, and the multiple-echo images were acquired within a breath-hold of approximately $13 \mathrm{~s}$.

\section{Performance Evaluations}

In the simulations, the estimated R2* and corresponding error maps were presented for visual inspection. The root mean square error (RMSE) was calculated as a quantitative criterion to quantify the accuracy of R2* mapping, and was defined as:

$$
R M S E=\sqrt{\frac{1}{M} \sum_{i}\left(R 2^{*}\left(x_{i}\right)-\widehat{R 2^{*}}\left(x_{i}\right)\right)^{2}},
$$


where $R 2^{*}\left(x_{i}\right)$ and $\widehat{R 2^{*}}\left(x_{i}\right)$ are the true and estimated $\mathrm{R} 2 *$ values at pixel $x_{i}$, respectively, and $M$ is the number of pixels in the selected ROI.

The mean and SD of the estimated R2* values in the liver parenchyma (1325 pixels) in the simulation study and those for each vial (340 pixels) in the phantom study were calculated for the quantitative performance assessment. Extreme outliers, which have distance to the interquartile range exceeding three times the length of the interquartile range, were excluded from the calculation. Considering the unknown ground truth, only the visual inspection of the quality of R $2 *$ maps was implemented for the in vivo study.

\section{RESULTS}

\section{Simulations}

Figure 2 shows the influence of smoothing parameter $h=\beta \sigma_{g}$ in the NLM-NLS and PCANR algorithms on R2* mapping. For both NLM-NLS and PCANR algorithms, the R2* mapping RMSEs follow a convex pattern with increasing $h$ from $0 \sigma_{g}$ to $7 \sigma_{g}$. The minimum RMSEs produced by the NLM-NLS and PCANR algorithms were substantially lower than that produced by the NLS algorithm for varying R2* values and noise levels. The PCANR algorithm consistently yields smaller minimum RMSEs than the NLM-NLS algorithm. In addition, the minimum RMSE of the PCANR algorithm is less sensitive to $h$ compared with that of the NLM-NLS algorithm. The PCANR algorithm with $h=2.0 \sigma_{g}$ is shown to approximately produce minimum RMSE for different R2* and SNR levels.

Figure 3 shows estimated R2* and corresponding error maps under varying SNR levels $(15,30$, and 60$)$ and $R 2 *$ reference values $\left(200,500\right.$, and $\left.800 \mathrm{~s}^{-1}\right)$. The NLS algorithm produced noisy R2* maps that exhibit a certain number of outliers, 
especially at the low SNR of 15. Although the NLM-NLS algorithm produced R2* maps that are less affected by the noise; however, measurement errors were still observed near the edges of liver parenchyma and vessels. Compared with the NLS and NLM-NLS algorithms, the PCANR algorithm effectively reduces the effect of noise on $\mathrm{R} 2 *$ mapping and produces accurate R2* maps in all cases, which can be observed clearly from the corresponding error maps (Fig. 3b).

The plots of R2* mapping RMSEs against R2* reference values under different SNRs $(15,30$, and 60) are shown in Figure 4. As partly shown in Figure 2, the RMSEs produced by the NLM-NLS and PCANR algorithms were substantially lower than that produced by the NLS algorithm. Compared with the NLM-NLS algorithm, the PCANR algorithm produced $46 \%-84 \%$ lower RMSEs. Figure 5 shows plots of mean and corresponding $\mathrm{SD}$ of parenchyma $\mathrm{R} 2 *$ values against $\mathrm{R} 2 *$ reference values under varying SNR levels $(15,30$, and 60). For low R2* values or high SNR levels, all three algorithms produced accurate R2* estimates that were close to the identity line. For high R2* values and low SNR levels, the mean of NLS and NLM-NLS measurements slightly overestimated the R2*, whereas the mean of PCANR measurements exhibited no bias from the R2* reference values. In addition, the PCANR algorithm produced the lowest SDs for any R2* reference value and SNR level, which is consistent with the performance of $\mathrm{R} 2 *$ mapping RMSEs (Fig. 4).

Figure 6 evaluates the performance of the NLM-NLS and PCANR algorithms under slowly-varying R2* values. Figure $6 \mathrm{~b}$ plots the RMSE of R2* mapping against smoothing parameter $h=\beta \sigma_{g}$. The PCANR algorithm with $h=2.0 \sigma_{g}$ also produces approximately minimum RMSEs that are well below the minimum RMSEs by the NLM-NLS algorithm. Figures $6 \mathrm{c}$ and $6 \mathrm{~d}$ present estimated R2* and corresponding error maps under varying SNR levels (15, 30, and 60). The PCANR results are less noisy than the NLM-NLS results, and with reduced errors. 


\section{Phantom Study}

Figure 7 shows the mean and corresponding SD of estimated R2* values in each vial depicted for datasets with different NEXs (1, 4, and 16). For the dataset with NEX of 1, the SNRs were from 22.24 to 55.62 for eight vials with different iron concentrations. For validation, the dataset with NEX of 16 was processed, and the decay signals in each vial were first averaged and then fitted to the $\mathrm{M}^{1} \mathrm{NCM}$ model. The resulting R2* values were highly correlated with the iron concentrations with an $r^{2}$ of 0.9996 and were used as the reference (slope of the reference line is 2.142). Similar to the previous results in the simulation study, the PCANR algorithm yields closer R2* estimates to the reference values than both the NLS and NLM-NLS algorithms. With regard to precision, the PCANR algorithm produces the smallest SD for each combination of iron concentration and NEX.

\section{In Vivo Study}

Figure 8 shows the in vivo R2* maps for four livers with none (Fig. 8a), mild (Fig. 8b), moderate (Fig. 8c), and severe iron overload (Fig. 8d). Similar to the results in the simulation study (Fig. 3), the R2* maps produced by the NLS algorithm were seriously degraded by the noise, and a certain number of extreme outliers exist in the severe iron-overloaded liver. The NLM-NLS algorithm led to blurring of tiny details (indicated by red arrows) and presence of residual noise near the edge of liver parenchyma (indicated by black arrows). Compared with the NLS and NLM-NLS algorithms, the PCANR algorithm effectively suppresses the effect of noise on R2* mapping and clearly preserves the tiny details. In addition, the distributions of parenchyma R2* values produced by the PCANR algorithm are more concentrated from the histograms (not shown) than those produced by the NLS and NLM-NLS algorithms. 


\section{DISCUSSION}

R2* mapping by conventional pixel-wise curve fitting is usually degraded by noise, especially when the SNR is low in high-speed or high-resolution acquisition. The PCANR algorithm improves the performance of liver R2* mapping by exploiting neighboring pixels to regularize the curve fitting for each target pixel. A distinct characteristic of the PCANR algorithm is that the regularization parameters are adaptively determined according to the inter-pixel signal similarity. Only those pixels with similar decay signals are assigned with large regularization parameters in the curve fitting for $\mathrm{R} 2 *$ mapping. The simulation, phantom, and in vivo results reveal that the PCANR algorithm can yield more accurate R2* maps than the NLS and NLM-NLS algorithms, especially for high R2* values under low SNR levels.

The PCANR algorithm reduces the effect of noise on R2* mapping by simultaneously fitting all the pixels similar to the target pixel, which is based on the assumption that the main difference between similar decay signals is caused by the noise. In this algorithm, all neighboring pixels contribute to the R2* quantification of each target pixel, and the pixels with larger regularization parameters contribute to the final R2* estimate. If the regularization parameters are set as zero for all neighboring pixels, the PCANR algorithm is reduced to the conventional NLS algorithm that independently fits each pixel. Note that the NLM-NLS algorithm also uses neighboring pixels to reduce the effect of noise on $\mathrm{R} 2 *$ mapping but in a two-step pattern: the serial images are first denoised, and pixel-wise curve fitting is then conducted for R2* mapping. However, this two-step pattern may induce the potential error propagation from the denoising step to the curve-fitting step. Compared with the NLM-NLS algorithm, the PCANR algorithm incorporates noise suppression and curve fitting into a unified one-step regularization framework, and this probably explains why the PCANR algorithm achieves better performance in reducing noise-related quantification errors for $\mathrm{R} 2 *$ mapping. 
Accurate R2* mapping can provide clinically relevant information that characterizes the spatial distribution of iron in the liver. In the practice of R2* relaxometry liver iron measurement, the $\mathrm{R} 2 *$ map produced by the NLS algorithm is usually degraded by noise, and thus cannot reveal the actual iron distribution in the liver, especially in the presence of severe iron overload. In addition, the mean of R2* measurements within an ROI by the NLS algorithm may overestimate iron level as shown by the simulation study. This overestimation is caused by the non-Gaussian distribution of fitted R2* values, as demonstrated in our previous work (29). The NLS algorithm also produces a certain number of extremely high $\mathrm{R} 2 *$ values above $2500 \mathrm{~s}^{-1}$. In the report of the representative R2* value in an ROI, no consensus was formed on whether to exclude those extremely high R2* values from the final measurement. These extremely high $\mathrm{R} 2 *$ values are successfully eliminated by the proposed PCANR algorithm. As a result, the reliability of $\mathrm{R} 2 *$ analysis is increased, especially for liver with severe iron overload. The blurring of tiny details in the NLM-NLS results (Fig. 8) is probably because no patches can be found truly similar to the central patch in the NLM denoising.

The performance of both PCANR and NLM-NLS algorithms depends on the smoothing parameter as shown in Figures 2 and $6 \mathrm{~b}$. The smoothing parameter $h$ controls the decay of the regularization parameters as a function of the Euclidean distance between the signals. A high $h$ leads to the over-smoothing in R2* map, whereas a low $h$ leads to the insufficient regularization from the neighboring pixels and results in noisy R2* map. As a widely-adopted approach $(20,21)$, the smoothing parameter $h=\beta \sigma_{g}$ was assessed using RMSEs in the simulation study. The results (Figs. 2 and 6b) reveal that the PCANR algorithm can consistently produce smaller minimum RMSEs at varying SNR levels and is less sensitive to the smoothing parameter than the NLM-NLS algorithm. This is because the PCANR algorithm actually combines denoising and the NLS fitting using a unified objective function, and is thus more tolerable to denoising errors than the NLM-NLS algorithm. The 
PCANR algorithm with a fixed smoothing parameter $h$ of $2.0 \sigma_{g}$ outperforms the NLM-NLS algorithm with its optimal smoothing parameter settings.

The performance of both PCANR and NLM-NLS algorithms also depends on the size of search window. Increasing the size of search window has the potential to improve the R2* mapping accuracy but at the expense of increased computational burden. In this work, the search window of $11 \times 11$ was experientially determined by balancing the accuracy and time cost. The computation time of the current implementation was $259 \mathrm{~s}$ on a 64-bit Windows 10 system with a $2.50 \mathrm{GHz}$ Intel Core processor and 16.0 GB of random access memory. Further increasing window size did not significantly improve R2* mapping accuracy. Note that the code was programmed using MATLAB (MATLAB 7.12.0, Mathworks) and the implementation can be further accelerated by $\mathrm{C}++$ programming and parallel computing, which is beyond the scope of this manuscript but warranted in a future study.

Simulation with fixed relaxation rate has the advantage of providing the reference $\mathrm{R} 2 *$ values for comparison and is consistent with the phantom experiment. In practice, the iron concentration might vary across the liver. Thus, we also performed simulation with a non-uniform $\mathrm{R} 2 *$ distribution by modeling the spatial distribution of $\mathrm{R} 2 *$ in the liver as a slowly-varying Gaussian function. Simulation with a R2* distribution closer to the realistic situation or a more comprehensive phantom development is warranted in future. The in vivo study is limited due to lack of reference standard for the algorithm evaluation. Although multiple averages can be used to improve the imaging, the substantially increased scanning time will increase the susceptibility to respiration motion. Future studies should make a concerted effort between scientists and clinicians to validate the development on large patient cohorts and to explore its clinical usefulness. 


\section{CONCLUSIONS}

Liver R2* mapping by conventional pixel-wise curve fitting is usually degraded by the noise, especially in the presence of severe iron overload. The PCANR algorithm can reduce the effect of noise on R2* mapping with improved accuracy and will benefit the assessment of hepatic iron and its distribution. Finally, the PCANR algorithm can be extended to the quantitative mappings of other MR parameters, such as $\mathrm{T} 1, \mathrm{~T} 2$, and diffusion coefficients. 


\section{FIGURE LEGENDS}

Figure 1. Liver mask, $S_{0}$ map, and examples of R2* map for simulations, as well as example of regularization parameters and decay signals in the PCANR algorithm. (a-b): Mask and $S_{0}$ map are obtained from an in vivo dataset. (c): An example of synthesized R2* map with R2* values of $500 \mathrm{~s}^{-1}$ for the parenchyma and $33 \mathrm{~s}^{-1}$ for blood vessels. $(\mathbf{d})$ : For pixels in a search window with size of $11 \times 11$ (red frame centered around a parenchyma pixel, which was marked by a green circle in (a)), regularization parameters are adaptively calculated according to Eq. [4] in the PCANR algorithm. (e): Discrete decay signals for pixels in the search window are plotted and marked by circles (by cross for the target pixel) with color according to their regularization parameters in $(\mathbf{d})$.

Figure 2. Influence of the smoothing parameter $h=\beta \sigma_{g}$ on R2* mapping RMSEs for different combinations of SNRs $(15,30$, and 60$)$ and R2* reference values $(100-$ $1000 \mathrm{~s}^{-1}$ ). Stars, dotted lines, and solid lines represent RMSEs by the NLS, NLM-NLS, and PCANR algorithms. Note that colors encode different R2* reference values (100-1000 s ${ }^{-1}$ from red, pass through yellow, green, cyan, blue, and magenta).

Figure 3. Estimated R2* maps (a) and error maps (b) for simulations with different SNRs $\left(15,30\right.$, and 60) and R2* reference values $\left(200,500\right.$, and $\left.800 \mathrm{~s}^{-1}\right)$.

Figure 4. R2* mapping RMSEs by the NLS, NLM-NLS, and PCANR algorithms for $\mathrm{SNR}=15,30$, and 60 .

Figure 5. Mean and SD values of the estimated R2* in the simulation study for SNR $=15,30$, and 60 .

Figure 6. Simulation with nonuniform R2* distribution. (a): The synthesized R2* map. Mean R2* value in the liver parenchyma was $800 \pm 160 \mathrm{~s}^{-1}$; the vessels have a 
constant R2* value of $33 \mathrm{~s}^{-1}$. (b): Influence of the smoothing parameter $h=\beta \sigma_{g}$ on R2* mapping RMSEs under different noise levels. (c-d): Estimated R2* maps and error maps. The RMSE was provided in the bottom-right corner of each R2* map.

Figure 7. Mean and SD values of the estimated R2* in the phantom study for NEX $=$ 1,4 , and 16. Note that for validation, the dataset with NEX of 16 is processed, the decay signals in each vial are first averaged and then fitted to the $\mathrm{M}^{1} \mathrm{NCM}$ model, and the resulting $\mathrm{R} 2 *$ values are linearly correlated with iron concentrations and used as reference.

Figure 8. In vivo R2* maps for livers with none (a), mild (b), moderate (c), and severe (d) iron overload. 


\section{REFERENCES}

1. Maria Domenica Cappellini, Alan Cohen, John Porter, Ali Taher, Viprakasit V. Guidelines for the Management of Transfusion Dependent Thalassaemia (TDT): Thalassaemia International Federation; 2014.

2. Angelucci E, Brittenham GM, McLaren CE, Ripalti M, Baronciani D, Giardini C, Galimberti M, Polchi P, Lucarelli G. Hepatic iron concentration and total body iron stores in thalassemia major. N Engl J Med 2000;343(5):327-331.

3. Peng P, Huang Z, Long L, Zhao F, Li C, Li W, He T. Liver iron quantification by 3 tesla MRI: calibration on a rabbit model. J Magn Reson Imaging 2013;38(6):1585-1590.

4. Perrault J, McGill DB, Ott BJ, Taylor WF. Liver biopsy: complications in 1000 inpatients and outpatients. Gastroenterology 1978;74(1):103-106.

5. Bravo AA, Sheth SG, Chopra S. Liver biopsy. N Engl J Med 2001;344(7):495-500.

6. Regev A, Berho M, Jeffers LJ, Milikowski C, Molina EG, Pyrsopoulos NT, Feng ZZ, Reddy KR, Schiff ER. Sampling error and intraobserver variation in liver biopsy in patients with chronic HCV infection. Am J Gastroenterol 2002;97(10):2614-2618.

7. Maharaj B, Maharaj RJ, Leary WP, Cooppan RM, Naran AD, Pirie D, Pudifin DJ. Sampling variability and its influence on the diagnostic yield of percutaneous needle biopsy of the liver. Lancet 1986;1(8480):523-525.

8. Ratziu V, Charlotte F, Heurtier A, Gombert S, Giral P, Bruckert E, Grimaldi A, Capron F, Poynard T, Group LS. Sampling variability of liver biopsy in nonalcoholic fatty liver disease. Gastroenterology 2005;128(7):1898-1906.

9. Vuppalanchi R, Unalp A, Van Natta ML, Cummings OW, Sandrasegaran KE, Hameed T, Tonascia J, Chalasani N. Effects of liver biopsy sample length and number of readings on sampling variability in nonalcoholic Fatty liver disease. Clin Gastroenterol Hepatol 2009;7(4):481-486.

10. Yokoo T, Browning JD. Fat and iron quantification in the liver: past, present, and future. Top Magn Reson Imaging 2014;23(2):73-94.

11. Hernando D, Levin YS, Sirlin CB, Reeder SB. Quantification of liver iron with MRI: state of the art and remaining challenges. J Magn Reson Imaging 2014;40(5):1003-1021.

12. Wood JC, Enriquez C, Ghugre N, Tyzka JM, Carson S, Nelson MD, Coates TD. MRI R2 and R2* mapping accurately estimates hepatic iron concentration in transfusion-dependent thalassemia and sickle cell disease patients. Blood 2005;106(4):1460-1465.

13. St Pierre TG, Clark PR, Chua-anusorn W, Fleming AJ, Jeffrey GP, Olynyk JK, Pootrakul P, Robins $E$, Lindeman R. Noninvasive measurement and imaging of liver iron concentrations using proton magnetic resonance. Blood 2005;105(2):855-861.

14. Meloni A, Zmyewski H, Rienhoff HY, Jr., Jones A, Pepe A, Lombardi M, Wood JC. Fast approximation to pixelwise relaxivity maps: validation in iron overloaded subjects. Magn Reson Imaging 2013;31(7):1074-1080.

15. Clark PR, St Pierre TG. Quantitative mapping of transverse relaxivity (1/T2) in hepatic iron overload: a single spin-echo imaging methodology. Magn Reson Imaging 2000;18(4):431-438.

16. Constantinides CD, Atalar E, McVeigh ER. Signal-to-noise measurements in magnitude images from NMR phased arrays. Magn Reson Med 1997;38(5):852-857.

17. Raya JG, Dietrich O, Horng A, Weber J, Reiser MF, Glaser C. T2 measurement in articular 
cartilage: impact of the fitting method on accuracy and precision at low SNR. Magn Reson Med 2010;63(1):181-193.

18. Feng Y, He T, Gatehouse PD, Li X, Harith Alam M, Pennell DJ, Chen W, Firmin DN. Improved MRI R2 * relaxometry of iron-loaded liver with noise correction. Magn Reson Med 2013;70(6):1765-1774.

19. Wang C, He T, Liu X, Zhong S, Chen W, Feng Y. Rapid look-up table method for noise-corrected curve fitting in the R2* mapping of iron loaded liver. Magn Reson Med 2015;73(2):865-871.

20. Zhao B, Lu W, Hitchens TK, Lam F, Ho C, Liang ZP. Accelerated MR parameter mapping with low-rank and sparsity constraints. Magn Reson Med 2015;74(2):489-498.

21. Zhang T, Pauly JM, Levesque IR. Accelerating parameter mapping with a locally low rank constraint. Magn Reson Med 2015;73(2):655-661.

22. Bhave $S$, Lingala SG, Johnson CP, Magnotta VA, Jacob M. Accelerated whole-brain multi-parameter mapping using blind compressed sensing. Magn Reson Med 2016;75(3):1175-1186.

23. Buades A, Coll B, Morel JM. A Review of Image Denoising Algorithms, with a New One. Multiscale Modeling \& Simulation 2005;4(2):490-530.

24. Feng $\mathrm{Y}$, He T, Feng $\mathrm{M}$, Carpenter JP, Greiser A, Xin X, Chen W, Pennell DJ, Yang GZ, Firmin DN. Improved pixel-by-pixel MRI R2* relaxometry by nonlocal means. Magn Reson Med 2014;72(1):260-268.

25. Wang C, Zhang X, Ma Y, Liu X, Hernando D, Reeder S, Chen W, Feng Y. A Noise Correction Model Incorporating Weighted Neighborhood Information for Liver R2* Mapping. In Proceedings of the 24th Annual Meeting of ISMRM, Singapore, 2016. p. 1914.

26. Levenberg K. A method for the solution of certain problems in least squares. Quart Appl Math 1944;2:164-168.

27. Marquardt DW. An algorithm for least-squares estimation of nonlinear parameters. Siam J Appl Math 1963;11(2):431-441.

28. Feng $\mathrm{Y}$, Feng $\mathrm{M}$, Gao $\mathrm{H}$, Zhang $\mathrm{X}$, Xin $\mathrm{X}$, Feng $\mathrm{Q}$, Chen $\mathrm{W}$, He $\mathrm{T}$. A novel semiautomatic parenchyma extraction method for improved MRI R2* relaxometry of iron loaded liver. J Magn Reson Imaging 2014;40(1):67-78.

29. Feng $\mathrm{M}$, Zhang $\mathrm{X}$, Gao H, Xu Y, Lin B, Liao J, Chen W, Feng Y. Optimal region-of-interest MRI R2* measurements for the assessment of hepatic iron content in thalassaemia major. Magn Reson Imaging 2014;32(6):647-653. 
(a)

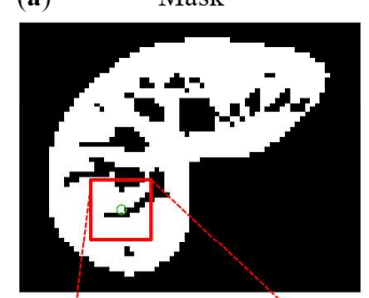

(d)

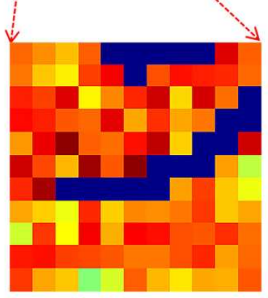

(b)
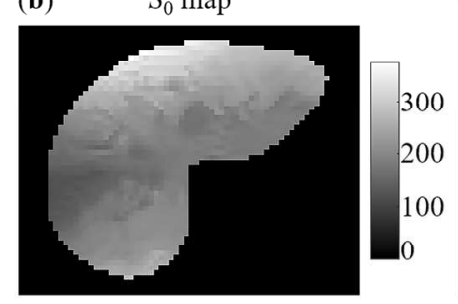

(c) R2* map

(e)

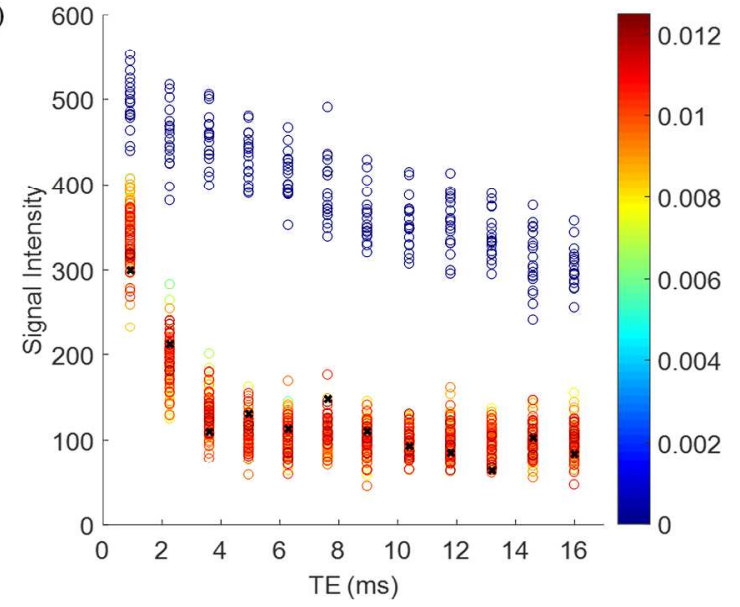

Figure 1. Liver mask, $S_{0}$ map, and examples of R2* map for simulations, as well as example of regularization parameters and decay signals in the PCANR algorithm. (a-b): Mask and $S_{0}$ map are obtained

from an in vivo dataset. (c): An example of synthesized R2* map with R2* values of $500 \mathrm{~s}^{-1}$ for the parenchyma and $33 \mathrm{~s}^{-1}$ for blood vessels. (d): For pixels in a search window with size of $11 \times 11$ (red frame centered around a parenchyma pixel, which was marked by a green circle in (a)), regularization parameters are adaptively calculated according to Eq. [4] in the PCANR algorithm. (e): Discrete decay signals for pixels in the search window are plotted and marked by circles (by cross for the target pixel) with color according to their regularization parameters in $(\mathbf{d})$.

$129 \times 95 \mathrm{~mm}(600 \times 600 \mathrm{DPI})$ 

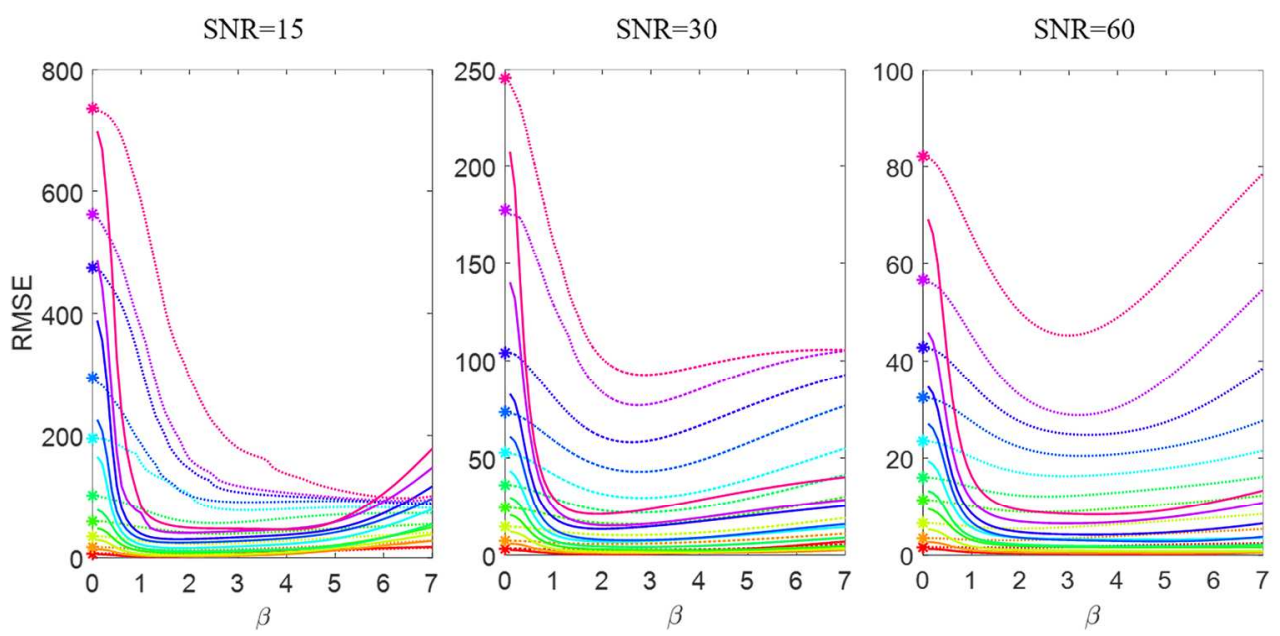

Figure 2. Influence of the smoothing parameter $h=\beta \sigma_{\mathrm{g}}$ on R2* mapping RMSEs for different combinations of SNRs $(15,30$, and 60$)$ and $R 2 *$ reference values $\left(100-1000 \mathrm{~s}^{-1}\right)$. Stars, dotted lines, and solid lines represent RMSEs by the NLS, NLM-NLS, and PCANR algorithms. Note that colors encode different R2* reference values (100-1000 s${ }^{-1}$ from red, pass through yellow, green, cyan, blue, and magenta).

$84 \times 40 \mathrm{~mm}(600 \times 600 \mathrm{DPI})$ 
(a) R2* maps
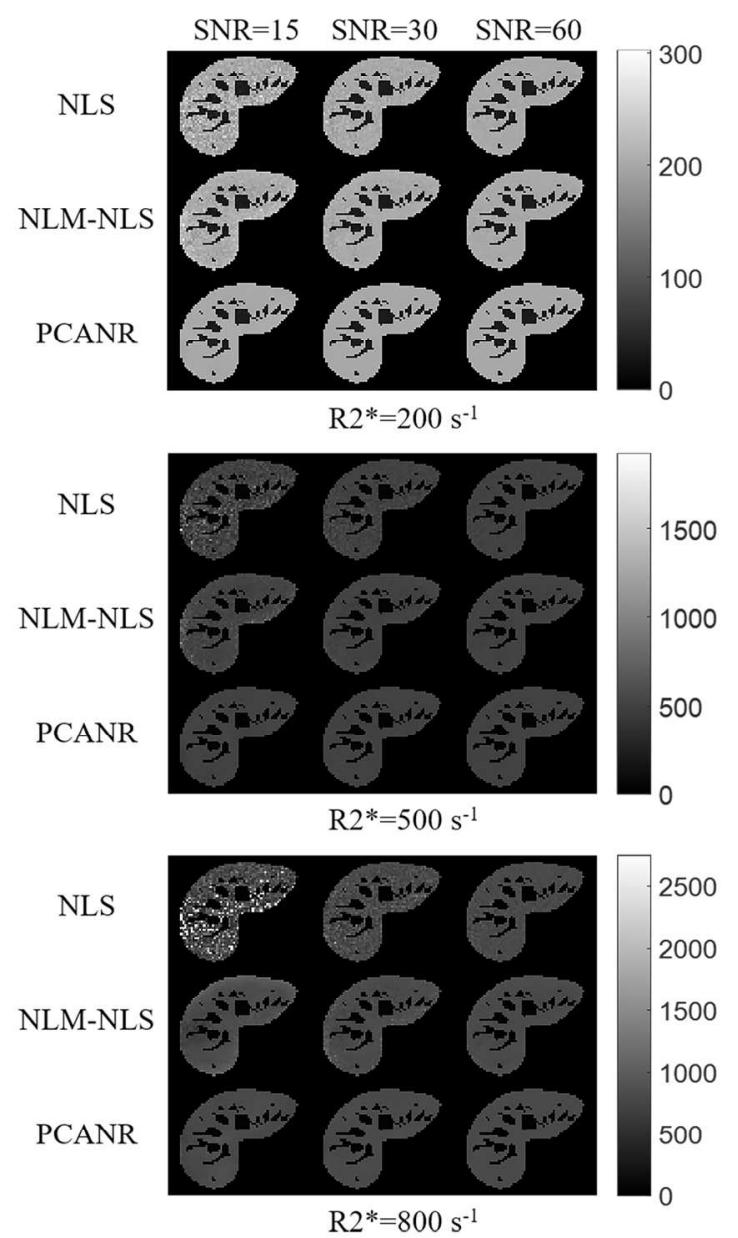

(b) Error maps
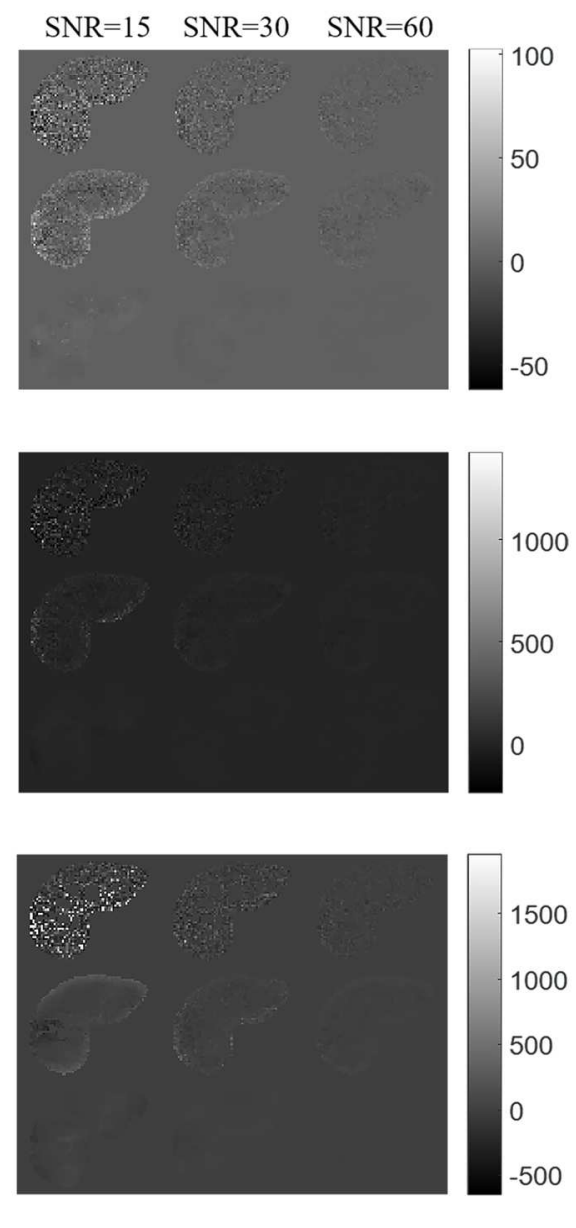

Figure 3. Estimated R2* maps (a) and error maps (b) for simulations with different SNRs (15, 30, and 60) and $R_{2} *$ reference values $\left(200,500\right.$, and $\left.800 \mathrm{~s}^{-1}\right)$.

$176 \times 178 \mathrm{~mm}(300 \times 300 \mathrm{DPI})$ 


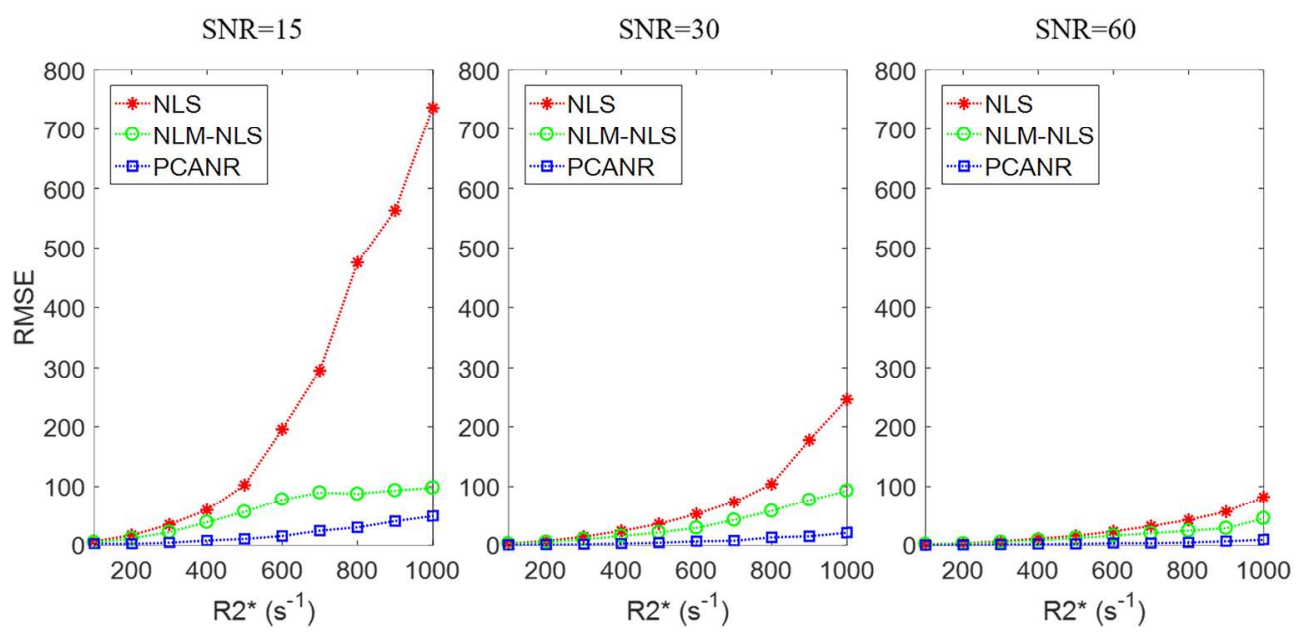

Figure 4. R2* mapping RMSEs by the NLS, NLM-NLS, and PCANR algorithms for SNR $=15,30$, and 60 .

$84 \times 40 \mathrm{~mm}(600 \times 600 \mathrm{DPI})$ 

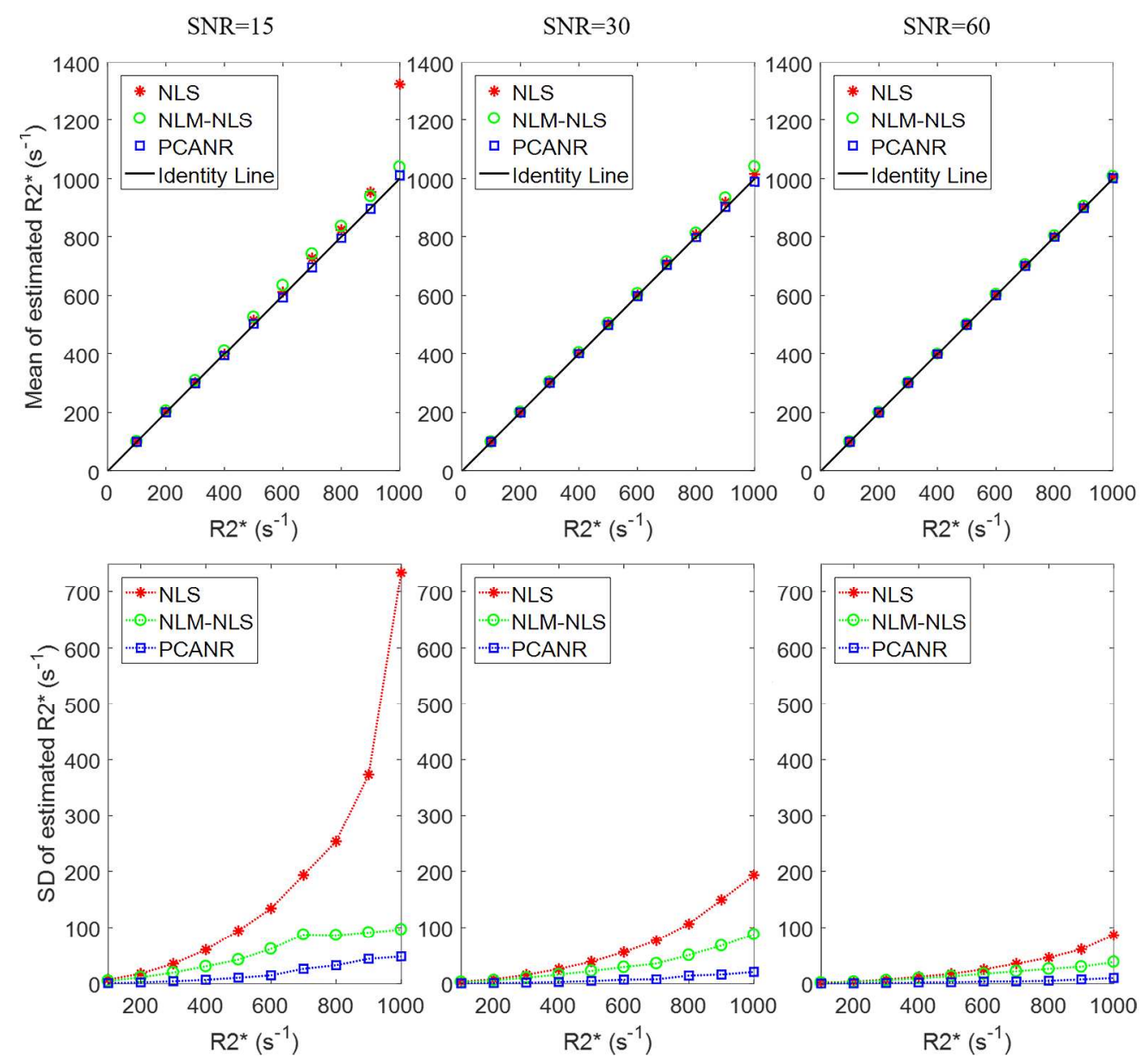

Figure 5. Mean and SD values of the estimated R2* in the simulation study for SNR $=15,30$, and 60 .

$164 \times 154 \mathrm{~mm}(600 \times 600$ DPI $)$ 
(a) Synthesized R2* map

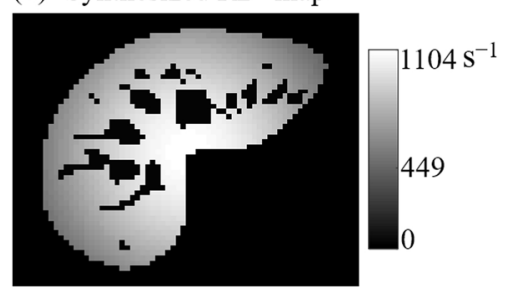

(b)

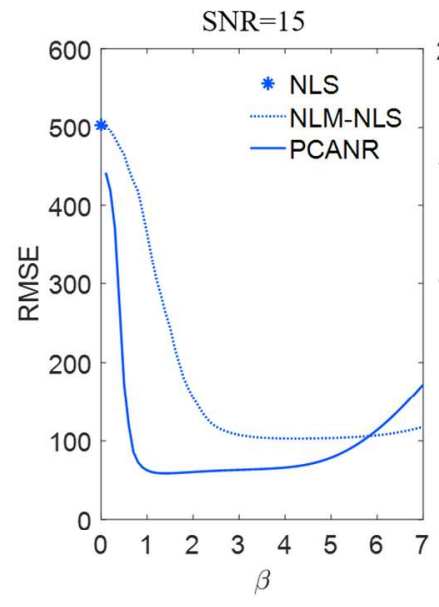

(c) R2* maps

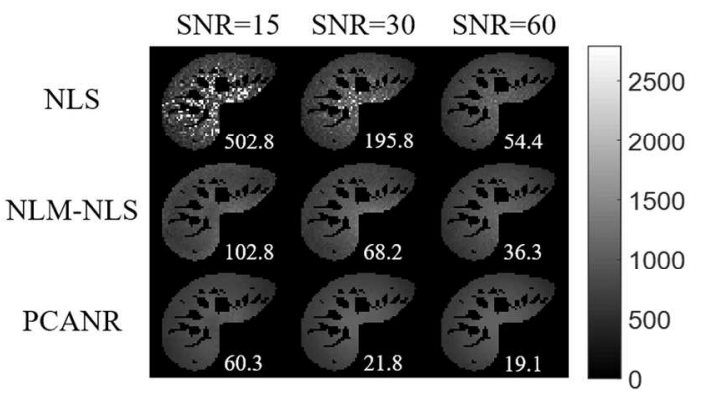

$\mathrm{SNR}=30$

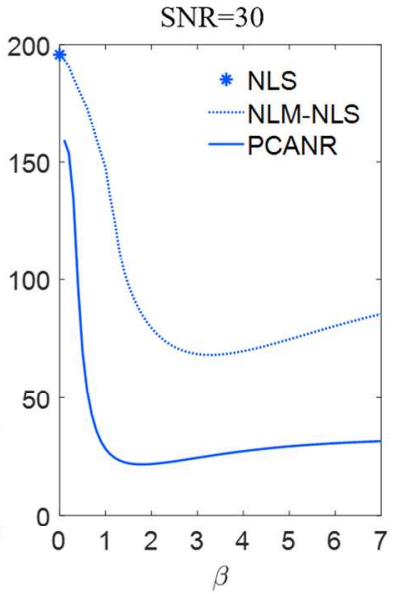

(d) Error maps
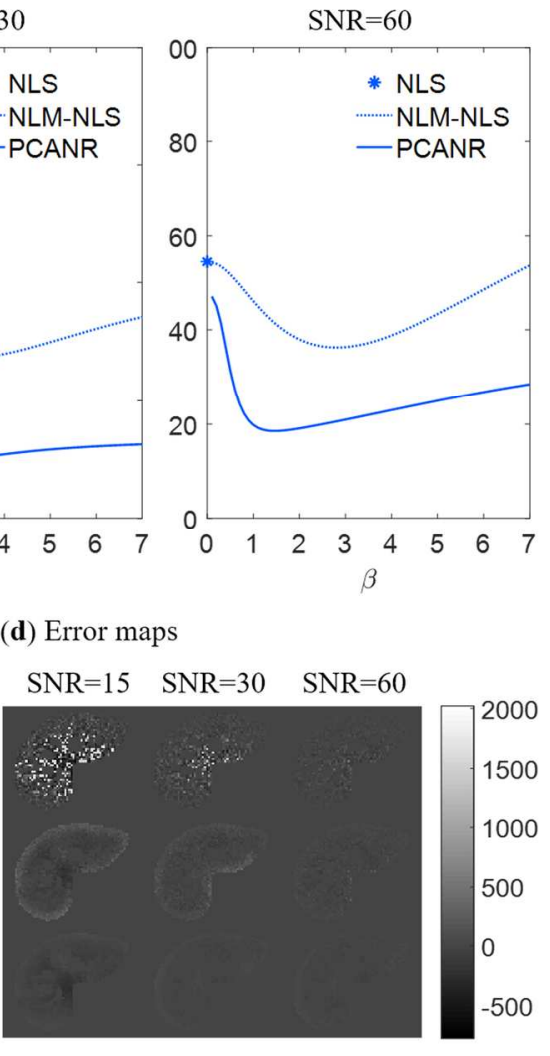

Figure 6. Simulation with nonuniform R2* distribution. (a): The synthesized R2* map. Mean R2* value in the liver parenchyma was $800 \pm 160 \mathrm{~s}^{-1}$; the vessels have a constant R2* value of $33 \mathrm{~s}^{-1}$. (b): Influence of the smoothing parameter $\mathrm{h}=\beta \sigma_{\mathrm{g}}$ on R2* mapping RMSEs under different noise levels. (c-d): Estimated R2* maps and error maps. The RMSE was provided in the bottom-right corner of each R2* map.

$197 \times 222 \mathrm{~mm}(300 \times 300 \mathrm{DPI})$ 

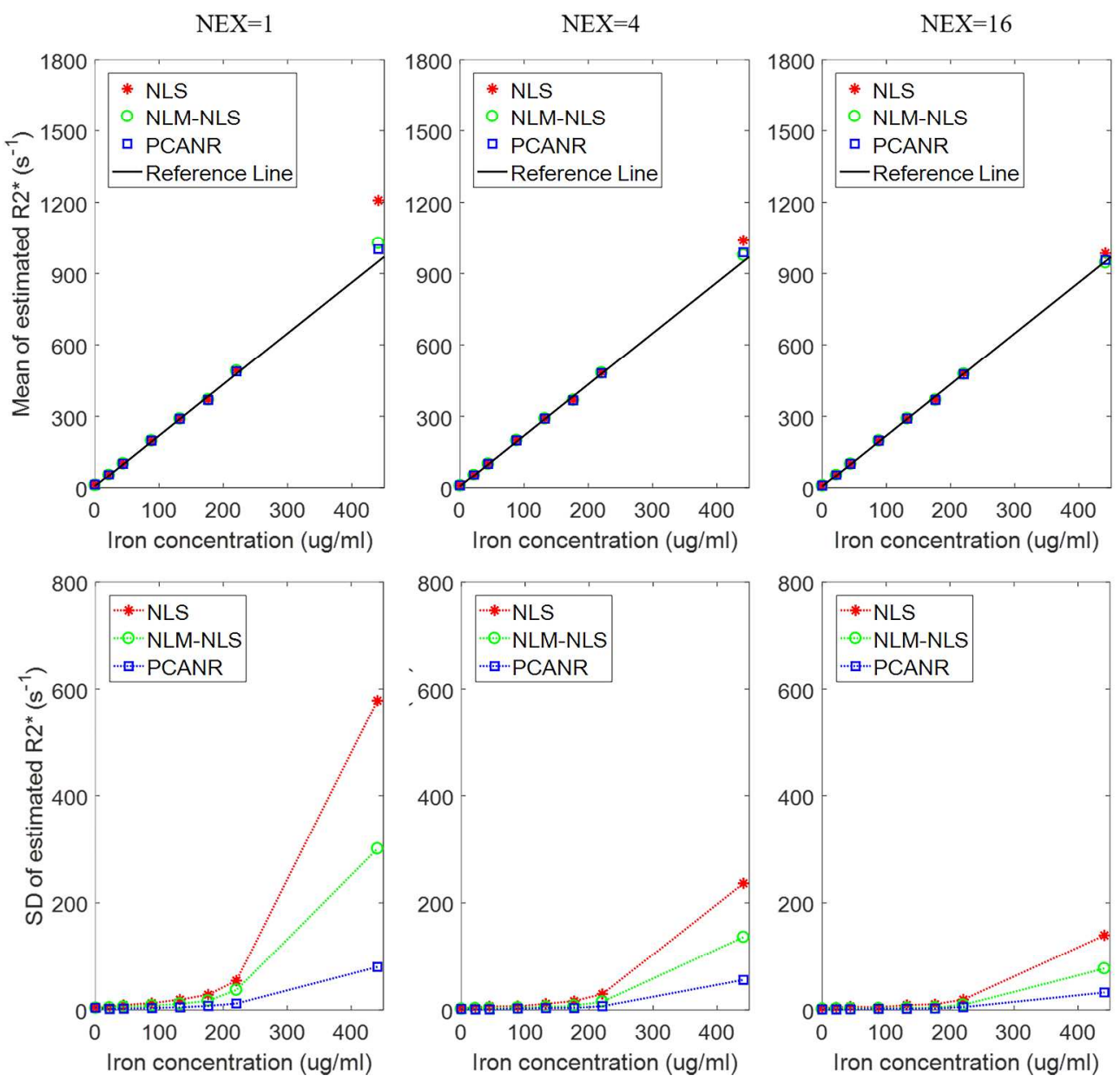

Figure 7. Mean and SD values of the estimated R2* in the phantom study for NEX $=1,4$, and 16 . Note that for validation, the dataset with NEX of 16 is processed, the decay signals in each vial are first averaged and then fitted to the $\mathrm{M}^{1} \mathrm{NCM}$ model, and the resulting $\mathrm{R} 2 *$ values are linearly correlated with iron concentrations and used as reference.

$166 \times 158 \mathrm{~mm}(600 \times 600 \mathrm{DPI})$ 
(a)

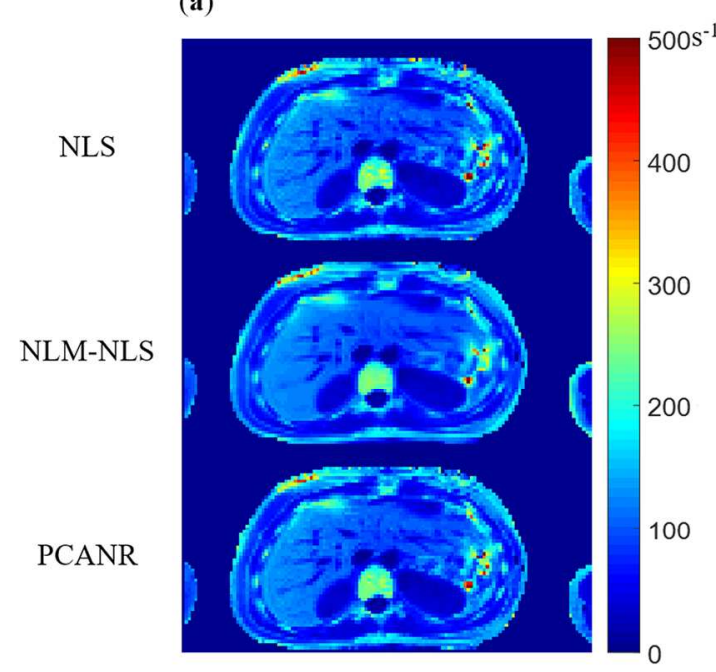

(c)

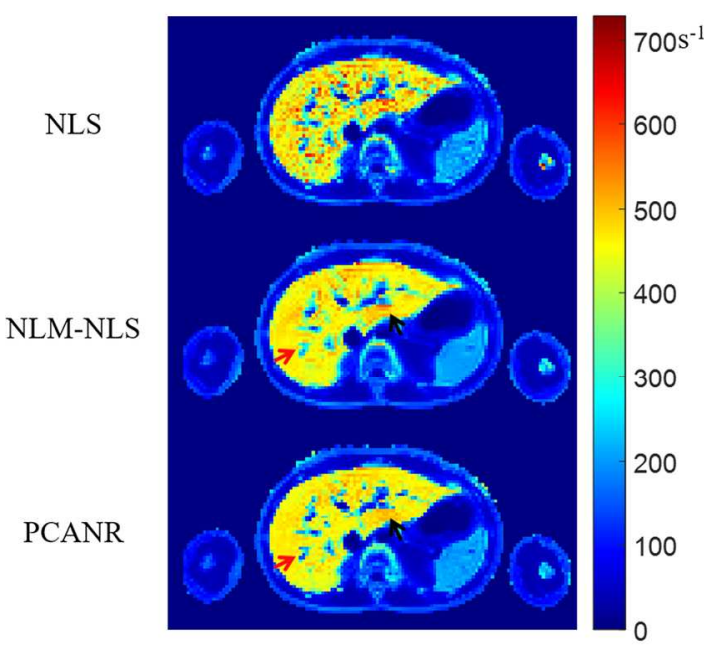

(b)

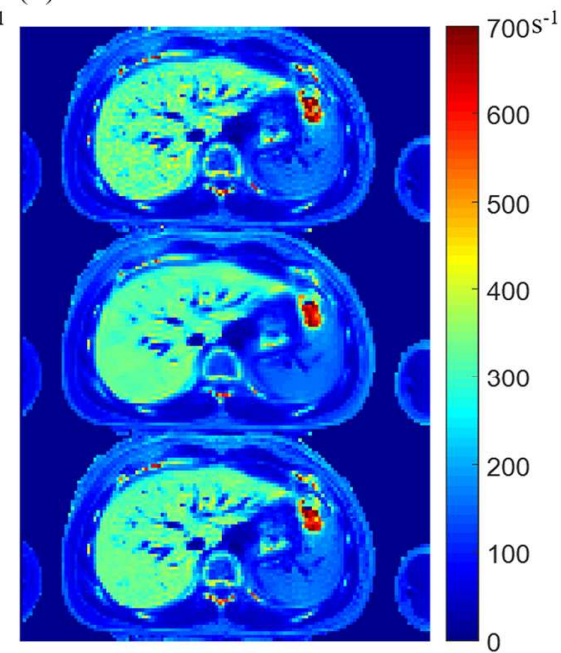

(d)

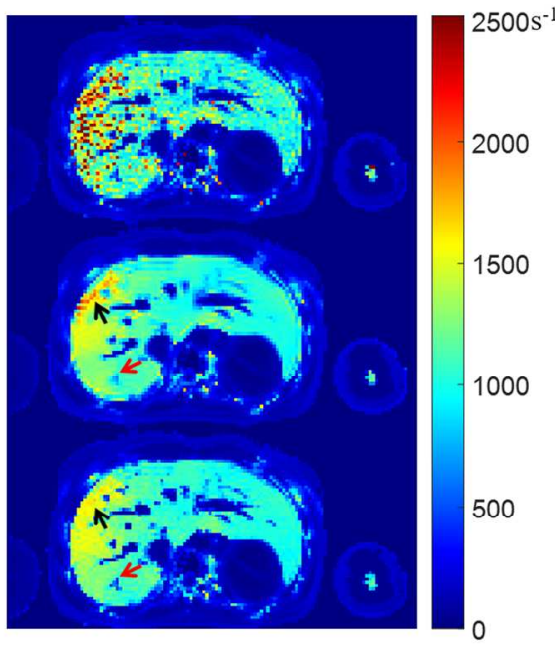

Figure 8. In vivo R2* maps for livers with none (a), mild (b), moderate (c), and severe (d) iron overload. $175 \times 187 \mathrm{~mm}(300 \times 300 \mathrm{DPI})$ 